\title{
Validación de la Escala de Apreciación de Agencia de Autocuidado (ASA) para Costa Rica, para población con enfermedad crónica ${ }^{1}$
}

Viriam Leiva Díaz ${ }^{2}$, Kenneth Cubillo Vargas ${ }^{3}$,Yuliany Porras Gutiérrez ${ }^{4}$, Tatiana Ramírez Villegas ${ }^{5}$, Isabel Sirias Wong ${ }^{6}$ Institución: Universidad de Costa Rica.

\section{COMO CITAR}

Leiva, V., Cubillo, K., Porras, Y., Ramírez, T., Sirias, I. (2016). Validación de la Escala de Apreciación de Agencia de Autocuidado (ASA) para Costa Rica, para población con enfermedad crónica. Rev. Enfermería Actual en Costa Rica, 31, 1-18. DOI:http://dx.doi.org/10.15517/revenf.v0i31.25486

\section{RESUMEN}

Introducción. Este artículo presenta los resultados del proceso de validación de apariencia, contenido y consistencia interna de la segunda versión en español de la escala ASA con población adulta costarricense con enfermedad crónica. La teoría de autocuidado desarrollada por Dorothea Orem (1983) acuña el término de la agencia de autocuidado, definida como: "la capacidad de participar en el autocuidado" (p.112), el cual ha sido el fundamento de instrumentos a nivel internacional como la escala de Ejercicio de Agencia de Autocuidado.

Método. Fue un estudio descriptivo, cuantitativo y psicométrico, realizado con una muestra de 106 personas.

Resultado. En la prueba de Kaiser-Meyer-Olkin (KMO) dio un índice de 0,65 dato aceptable; la prueba de esfericidad de Bartlett resultó estadísticamente significativa con un valor de p<0,000; 276 gl y un Chi ${ }^{2}$ de 563,9. De igual forma el estudio factorial mediante el método ACP con rotación Varimax sugirió el análisis con 5 ejes factoriales que explicaron el 56,76\% de la varianza total. El Alfa de Cronbach fue 0,84 (84\%) lo que significa que el total de los ítems que contempla la escala logran medir un mismo concepto o se correlacionan positivamente.

Conclusión: Se logró afirmar que el instrumento cuenta con una adecuada validez de contenido, con propiedades psicométricas válidas y adecuadas, además se demostró una alta confiabilidad.

Palabras claves: consistencia-interna, enfermedad crónica, validación-de-escala

\footnotetext{
${ }^{1}$ Fecha de recibido: 17 de marzo de 2015

Fecha de aceptación: 3 de diciembre de 2015

${ }^{2}$ Enfermera. Profesora, investigadora. Escuela de Enfermería de la Universidad de Costa Rica. Correo electrónico: viriaml@gmail.com

${ }^{3}$ Enfermero. Profesor. Escuela de Enfermería de la Universidad de Costa Rica. Correo electrónico: kenncub@hotmail.com

${ }^{4}$ Enfermera. Trabajo independiente. Costa Rica. Correo electrónico: yulianyp@gmail.com

${ }^{5}$ Enfermera. Hospital CIMA San José. Costa Rica. Correo electrónico: tatyanra@yahoo.es

${ }^{6}$ Enfermera. Trabajo independiente. Costa Rica. Correo electrónico: jsw87@,hotmail.com
} 


\section{Validation of the scale Appreciation of Self Care Agency (ASA) for Costa Rica, for people with chronic disease ${ }^{1}$}

Viriam Leiva Díaz ${ }^{2}$, Kenneth Cubillo Vargas ${ }^{3}$, Yuliany Porras Gutiérrez ${ }^{4}$, Tatiana Ramírez Villegas ${ }^{5}$, Isabel Sirias Wong ${ }^{6}$

Institution: University of Costa Rica.

\section{CITED}

Leiva, V., Cubillo, K., Porras, Y., Ramírez, T., Sirias, I. (2016). Validation of the scale Appreciation of Self Care Agency (ASA) for Costa Rica, for people with chronic disease. Rev. Enfermeria Actual en Costa Rica, 31, 1-18. DOI: http://dx.doi.org/10.15517/revenf.v0i31.25486

\section{ABSTRACT}

Introduction. This paper presents the results of the validation process of appearance, content and internal consistency of the second Spanish version of the ASA scale with Costa Rican adult population with chronic disease. Self-care theory developed by Dorothea Orem (1983) coined the term self-care agency, defined as "the ability to engage in self-care" (p.112), which has been the foundation of international instruments as scale Exercise Self Care Agency.

Method. It was a descriptive study with a quantitative and psychometric approach, conducted with a sample of 106 people.

Result. The test Kaiser-Meyer-Olkin (KMO) gave acceptable data rate of 0.65; the Bartlett test of sphericity was statistically significant with a p-value $<0.000$; G1 276 and 563.9 chi. Likewise, the study by the ACP factorial method with Varimax rotation analysis suggested 5 factorial axes which accounted for $56.76 \%$ of the total variance. Cronbach's alpha was $0.84(84 \%)$, which means that the total of the items that fail to measure the scale includes the same concept, or positively correlated.

Conclusion. It managed to say that the instrument has adequate content validity, with valid and appropriate psychometric properties also demonstrated high reliability.

Key words: chronic-disease, internal-consistency, validation-of-scale.

\footnotetext{
${ }^{1}$ Date of receipt: March 17, 2014

Date of acceptance: December 3, 2015

${ }^{2}$ Nurse. Profesor. Researcher. School of Nursing, University of Costa Rica. E-mail: viriaml@gmail.com

${ }^{3}$ Nurse. Profesor. School of Nursing, University of Costa Rica. E-mail: kenncub@hotmail.com

${ }^{4}$ Nurse. Independent work. Costa Rica. E-mail: yulianyp@gmail.com

${ }^{5}$ Nurse. CIMA San José Hospital. Costa Rica. E-mail: tatyanra@yahoo.es

${ }^{6}$ Nurse. Independent work. Costa Rica. E-mail: jsw87@,hotmail.com
} 


\section{Validação da Escala de Avaliação da Agência Autocuidado (ASA) para a Costa Rica, para as pessoas com doenças crónicas ${ }^{1}$}

Viriam Leiva Díaz ${ }^{2}$, Kenneth Cubillo Vargas ${ }^{3}$, Yuliany Porras Gutiérrez ${ }^{4}$, Tatiana Ramírez Villegas ${ }^{5}$, Isabel Sirias Wong ${ }^{6}$

Instituição: Universidade de Costa Rica.

\section{CITAÇÃO}

Leiva, V., Cubillo, K., Porras, Y., Ramírez, T., Sirias, I. (2016). Validação da Escala de Avaliação da Agência Autocuidado (ASA) para a Costa Rica, para as pessoas com doenças crónicas. Rev. Enfermería Actual en Costa Rica, 31, 1-18. DOI: http://dx.doi.org/10.15517/revenf.v0i31.25486

\section{RESUMO}

Introdução. Este artigo apresenta os resultados do processo de validação de aparência, conteúdo e consistência interna da segunda versão espanhola da escala ASA com a população adulta da Costa Rica com a doença crônica. Teoria da auto-cuidado desenvolvido por Dorothea Orem (1983) cunhou a agência de autocuidado prazo, definida como "a capacidade de envolver-se em auto-cuidado" (p.112), que tem sido a base de instrumentos internacionais como dimensionar Agência Autocuidado Exercício.

Método. Foi um estudo descritivo, quantitativa e psicométrica, realizado com uma amostra de 106 pessoas.

Resultado. No teste de Kaiser-Meyer-Olkin (KMO) deu taxa de dados aceitável de 0,65; o teste de Bartlett de esfericidade foi estatisticamente significativa com um valor de $\mathrm{p}<0,000 ; 276 \mathrm{gl}$ e Chi2 de 563,9. Da mesma forma, o estudo realizado pelo método fatorial ACP com a análise rotação Varimax sugeriu 5 eixos fatoriais que responderam por $56,76 \%$ da variância total. alfa de Cronbach foi de $0,84(84 \%)$ o que significa que o total dos elementos que não conseguem medir a escala inclui o mesmo conceito ou positivamente correlacionados.

Conclusão. Conseguiu dizer que o instrumento tem validade de conteúdo adequado, com propriedades psicométricas válidas e adequadas também demonstrou alta confiabilidade.

Palavras-chave: consistência-interna, doença-crônica, validação-de-escala.

\footnotetext{
${ }^{1}$ Data de recebimento: 17 de março de 2014

Data de aceitação: 3 de dezembro de 2015

${ }^{2}$ Enfermeira. Professor, pesquisador. Escola de Enfermagem da Universidade de Costa Rica. Costa Rica. Correio eletrônico: viriaml@gmail.com

${ }^{3}$ Enfermeiro. Professor. Escola de Enfermagem da Universidade de Costa Rica. Costa Rica. Correio eletrônico: kenncub@hotmail.com

${ }^{4}$ Enfermeira. Trabalho independente. Costa Rica. Correio eletrônico: yulianyp@gmail.com

${ }^{5}$ Enfermeira. Hospital CIMA San José. Costa Rica. Correio eletrônico: tatyanra@yahoo.es

${ }^{6}$ Enfermeira. Trabajo independiente. Costa Rica. Correio eletrônico: jsw87@,hotmail.com
} 


\section{Revista Electrónica Enfermeria Actual en costa Rica}

\section{INTRODUCCIÓN}

La teoría de autocuidado desarrollada por Dorothea Orem (1983) acuña el término de la agencia de autocuidado, definida como: "la capacidad de participar en el autocuidado" (p.112), el cual ha sido el fundamento de instrumentos a nivel internacional como la escala de Ejercicio de Agencia de Autocuidado (ESCA), el Cuestionario de la Estructura Cognitiva relacionado con salud y autocuidado, y el cuestionario de percepción de la agencia de Autocuidado (PSCA) (Achury, Sepúlveda y Rodríguez, 2008); sin embargo, uno de los instrumentos que recoge, de forma más clara y concisa, los principales elementos de la capacidad de autocuidado a evaluar en las personas es el desarrollado en Holanda por Evers, Isenberg, Philipsen, Senten y Brouns (Rodríguez, Díaz, 2011), en 1983 denominado "Escala de Apreciación de Agencia de Autocuidado (ASA, por sus siglas en inglés, Appraisal of the Selfcare Agency).

Tres elementos fundamentales por desarrollar en la capacidad de autocuidado-que recoge la Escala de Apreciación de Agencia de Autocuidado (ASA)-, son las capacidades fundamentales y disposición de autocuidado, las capacidades para operaciones de autocuidado y los componentes de poder (Rivera, 2006; Rivera y Díaz, 2007). El último -los componentes de poder- se utilizó como fundamento para la escala ASA, el cual contempla diez componentes: máxima atención o vigilancia, control de energía física, control del movimiento del cuerpo, habilidad para razonar, motivación para la acción, habilidades para tomar decisiones, conocimiento, repertorio de habilidades, habilidades para ordenar las acciones de autocuidado y habilidades para integrar (Evers, 1998).

En el ámbito mundial, la escala ASA ha sido utilizada y traducida en varios idiomas en diversas regiones como son Europa (Suiza, Noruega, Dinamarca, España, Turquía,), Asia Oriental (Hong Kong). En América usada en varios países como Guatemala (Porras, 2002), Nicaragua, (Ortiz, 2002), Honduras (Monge 2004) y Colombia. (Rivera, 2006) (Manrique-Abril, Fernández y Velandia 2009); por lo que, como mencionan Velandia y Rivera (2009), fue necesario realizar la validez del constructo y se detectó, que la escala mostraba que el instrumento tenía un poder discriminatorio de los grupos según el nivel de dependencia o independencia.

Esta escala fue traducida al español en 1998 por Gallegos en México el cual poseía originalmente -en formato Likert- 5 alternativas de respuesta (totalmente en desacuerdo, en desacuerdo, indeciso, de acuerdo y totalmente de acuerdo); sin embargo, para ser utilizado en el contexto colombiano se le modificó a 4 alternativas al formato de: nunca, casi nunca, casi siempre y siempre y los ítems 4, 6, 13 y 16 fueron escritos nuevamente de manera más simple y gramaticalmente correctos (Velandia y Rivera, 2009) conservando el rango de 24 a 96 puntos.

En Colombia, respecto de la escala de ASA, Manrique-Abril, Fernández y Velandia (2009) realizaron el análisis factorial con población con patología crónica, delo que se obtuvo que poseía propiedades psicométricas validas, así como su confiabilidad, de tal manera que los 24 ítems medían un mismo concepto, lo cual le confirió consistencia interna (Velandia y Rivera, 2009).

Para desarrollar la validación de un instrumento se debe seguir una serie de pasos que fueron el objetivo de la investigación: desarrollar la validez de apariencia, la validez de contenido y la consistencia interna de la Escala de ASA en su segunda versión en español en Costa Rica. 


\section{Revista Electrónica Enfermeria Actual en costa Rica}

\section{MATERIALES Y MÉTODOS}

La investigación se enmarcó en un estudio de tipo descriptivo y transversal realizado durante el 2011, bajo un enfoque cuantitativo y con un diseño no experimental (Hernández, Fernández y Baptista, 2006), que a su vez fue psicométrico, pues pretendió la adaptación al contexto costarricense (Kerlinger y Lee, 2002).

- En la validez de apariencia, se requirió del criterio de cinco expertos (Contreras, Flórez y Herrera, 2008) de Enfermería, estadística, filología y psicología (específicamente en psicometría) y cinco personas ajenas a la población meta, pero que cumplían con los criterios de inclusión; se analizó los criterios aportados para determinar su pertinencia (Sánchez y Echeverri, 2004).

- En la validez de contenido inicialmente se determinó la viabilidad mediante la prueba de KaiserMeyer-Olkin (KMO) y la prueba de esfericidad de Barlett, (Lamprea y Gómez, 2007), luego se le aplicó el instrumento a la muestra y se procedió una matriz de correlación y el análisis factorial utilizando la técnica de extracción de componentes principales y la de rotación Varimax. (Morales, 2011).

- $\quad$ En el proceso de consistencia interna se procedió a estimar la confiabilidad de la escala por medio del índice estadístico de Alfa de Cronbach (George y Mallery, 1995).

La unidad de análisis fue la persona adulta, según los periodos de desarrollo, descritos por Papalia (2005) como etapa de la adultez temprana (20-40 años), intermedia (40-65 años) y tardía (65 años en adelante); las primeras dos etapas abarcadas por todos los funcionarios del INS Salud y la última, por la población adulta tardía del Programa Integral de la Persona Adulta Mayor (PIAM) de la Universidad de Costa Rica, matriculadas en el primer semestre del año 2011.

La muestra fue de tipo no probabilístico por cuotas (Hernández, Fernández y Baptista, 2006), constituida por 106 personas con algún tipo de enfermedad crónica. En este caso, se define salud según lo manifestado por la OMS (2008) que define las enfermedades crónicas como "enfermedades de larga duración y por lo general de progresión lenta" (s.p.). Dentro de este grupo se incluyen los diferentes tipos de cáncer, enfermedades respiratorias, enfermedades congénitas, así como las patologías de tipo cardiovascular como la hipertensión arterial y enfermedad cerebro-vascular.

El tamaño muestral se determinó considerando lo mencionado por Norman y Streiner (1996), citados por Sánchez y Echeverri (2004), según los cuales "para efectuar este tipo de análisis se requieren, por lo menos, cinco pacientes por cada ítem que tenga el instrumento, pero no menos de 100 pa $\neg$ cientes en total" (p8).

La recolección de los datos se llevó a cabo durante el año 2011, por medio de un cuestionario autoadministrado individual que constó de cuatro partes: la primera fue la guía para validación de jueces expertos (aplicada únicamente a los 5 expertos seleccionados), la segunda fue de datos sociodemográficos y de autocuidado por medio de preguntas abiertas y cerradas; la tercera fue la escala de Capacidad de Agencia de Autocuidado y la última, se destinó a recolectar la percepción personal acerca del formato, redacción y capacidad de comprensión de la escala por parte de los participantes.

Las variables consideradas responden a tres dimensiones: datos socio-demográficos que contempló: nacionalidad, sexo, edad, ocupación, lugar de residencia, grado de escolaridad, estado civil, ingresos económicos. 
La segunda se refiere al estado de salud, que incluyó enfermedad, tratamiento farmacológico, tratamiento no farmacológico, seguro de salud, mientras que la última dimensión abarcó las variables de: alimentación, actividad y descanso, soledad e interacción social, prevención de peligros contra la vida, el funcionamiento y bienestar personal y promoción del funcionamiento y desarrollo humano.

En cuanto a las consideraciones éticas, la investigación se basó en la autonomía, beneficencia y no maleficencia, se llevó a cabo el proceso de consentimiento informado -información, comprensión, aprobaciónasesoramiento y la presentación y divulgación de los resultados (Leiva, 2009).

Para analizar la información, una especialista en estadística de la Universidad de Costa Rica brindó asesoría; para procesarla, se utilizó el paquete estadístico CSPro versión 4.1 y el programa SPSS versión 18.0, para posteriormente exportar los datos al paquete Excel 2010.

\section{RESULTADOS}

Los resultados serán presentados según las unidades de análisis del apartado previo: se iniciará, con los aspectos sociodemográficos y de autocuidado.

\section{Aspectos socio-demográficos y de autocuidado}

En el aspecto de nacionalidad, de la totalidad de la población (106), 105 personas fueron costarricenses y una nicaragüense; un 41,5\% fueron hombres y un 58,5\%, mujeres. Ambos sexos se ubican según su edad en un $32,1 \%$ en la adultez temprana, un $37,7 \%$ en la adultez intermedia y un 30,2 \% en la adultez tardía; en cuanto al estado civil, hubo un predominio de personas casadas con un $50,9 \%$; un $22,6 \%$, solteras y un $10,4 \%$ de personas que enviudaron.

El aspecto de escolaridad reflejó que el grado universitario fue el que tuvo mayor representación con un $33,0 \%$ de universidad completa y un $14,2 \%$, de incompleta, seguido de un $13,2 \%$; en cuanto a la ocupación principal se demostró un predominio de ocupaciones de nivel profesional seguido del nivel técnico y en tercer lugar se ubicaron las personas que son amas de casa; un 7,5\% no respondió a qué se dedicaba. Respecto del salario o ingreso mensual de las personas, un 27,4\% afirmó recibir entre $\mathbb{C 5 0 0} 000$ y $\mathbb{C} 1000000$; un 24,5\%, entre $\not C 200000$ y $\not C 500$ 000; un 22,6\%, entre $\mathbb{C} 1000000$ o más, y un 18,9\%, menos de $\not C 200000$.

En cuanto al lugar de residencia, este se ubicó principalmente en la provincia de San José con un 71,7\% seguido de Heredia con un 11,3\% y en tercer lugar la provincia de Alajuela con un $8,5 \%$ de las personas. Por otro lado, en aspectos de autocuidado de las personas, de las enfermedades crónicas establecidas, como se evidencia en el gráfico siguiente, las personas indicaron que la hipertensión arterial ocupa el primer lugar, seguido de la diabetes mellitus y las enfermedades coronarias. 


\section{Grafico 1}

Distribución relativa de las respuestas de los entrevistados según enfermedades crónicas que padecen. Costa Rica, 2011

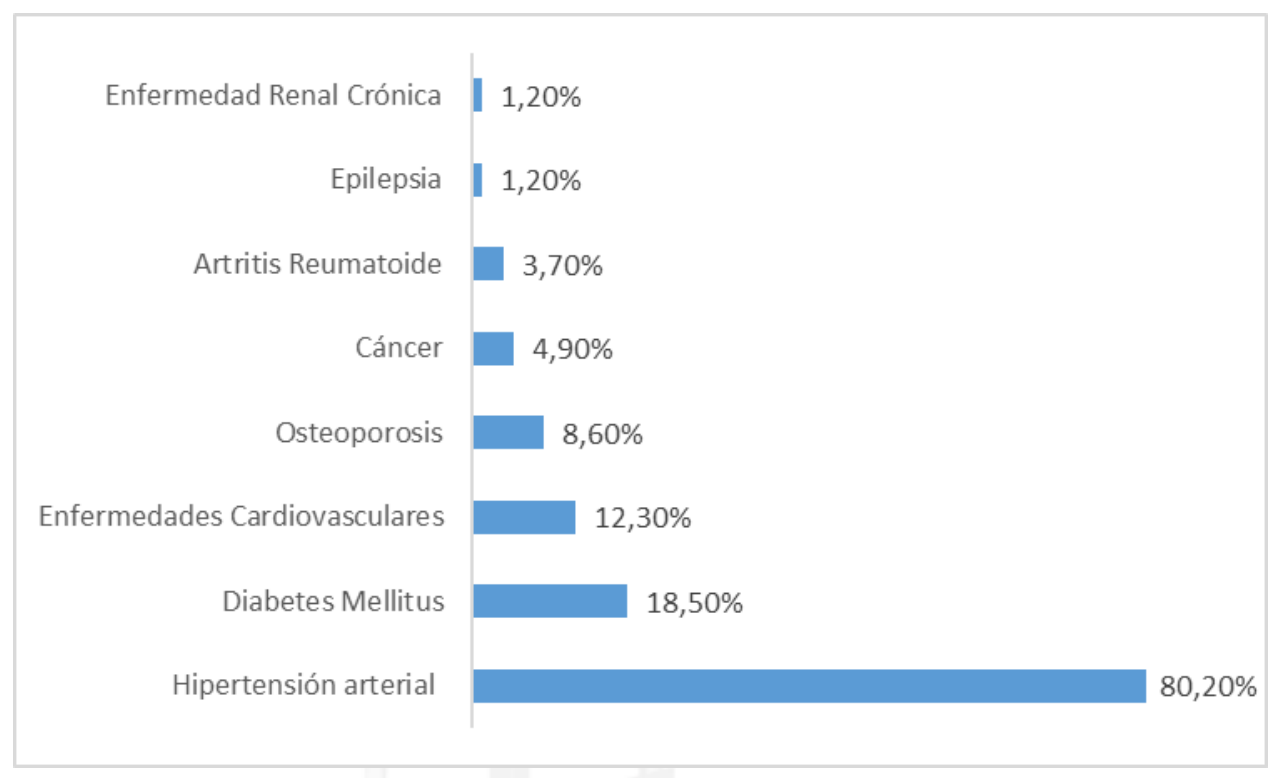

Fuente: Cubillo, Porras, Ramírez y Sirias 2011

Además de estas enfermedades, un 50,9\% de las personas indicó tener otra enfermedad concomitante, clasificadas en los siguientes grupos: en primer lugar se ubica los problemas metabólicos y genitourinarios (en un $5,7 \%$ de las personas), seguido de los problemas respiratorios, inmunológicos y de sentidos especiales (12,3\%); los problemas gastrointestinales y nerviosos ocuparon el tercer lugar (un 11,3\%) seguido de los problemas, cardiovasculares y hematológicos (un 6,6\%) y, por último, los problemas osteomusculares y articulares presentados $(0,9 \%)$.

En relación con el consumo de fármacos, el 88,7\% de la muestra indicó tomar alguno y; un 39,6\% de la muestra indicó llevar un tratamiento no farmacológico.

Dichos medicamentos correspondieron según su agrupación en un 51,9\% antihipertensivos y antiarrítmicos; un 15,1\%, hipoglucemiantes, hipolipemiantes antieméticos y antiulcerosos; un 4,7\% analgésicos, antiinflamatorios, antipiréticos y glucocorticoide $\mathrm{y}$, un 1,9\%, antidepresivos, anticonvulsionantes. Los tratamientos no farmacológicos correspondieron a un 13,2\% de hierbas y bebidas naturales: un $11,3 \%$, a productos comerciales y un $9,4 \%$ a no tener estrés, hacer ejercicio, llevar terapias especiales y otros.

Por otro lado, un 96,2\% afirma que cuenta con seguro de salud principalmente de la Caja Costarricense de Seguro Social, un 95,3\% asegura que asiste a centros de salud, público o privado, mientras que un 4,7\% restante refiere que no asiste, por razones como ser atleta $(3,8 \%)$ y porque hasta el momento su salud ha sido buena y no ha necesitado $(0,9 \%)$. 


\section{Revista Electrónica Enfermeria Actual en costa Rica}

\section{Validación de la Escala ASA}

- Validación de apariencia

\section{Modificaciones estructurales de la escala ASA}

Ítem 1. "A medida que cambian las circunstancias yo voy haciendo ajustes para mantener mi salud".

Dos de las expertas comentaron que, en cuanto a los criterios de claridad y suficiencia, la palabra "circunstancias" debía ser variada. Se analizó su conceptualización según el Diccionario de la Real Academia Española (2011) que la define como "dicho de una cosa que de algún modo está influida por una situación ocasional", por tanto, el término se ajusta a lo que el ítem pregunta; a su vez coincide con lo planteado por Orem (1983), cuando hace referencia a que el ser humano, por naturaleza, tiene necesidades comunes para crear y mantener condiciones de vida que sostengan los procesos vitales, así como el mantenimiento y promoción de su integridad funcional. Por tanto, considerando que el desarrollo humano en todas sus etapas, requiere el mantenimiento de condiciones que promuevan los procesos necesarios para el avance en cada etapa del ciclo vital, y que el autocuidado está dirigido a alcanzar objetivos en distintas circunstancias, necesariamente una serie de acciones hechas con consecuencia, se considera correctamente como un sistema de acción o un proceso dinámico que varía según las condiciones o circunstancias que se presenten día con día (Orem,1983), por lo que este ítem se mantuvo redactado de la misma manera.

Ítem 2. "Reviso si las formas que practico habitualmente para mantenerme con salud son buenas".

Una de las personas con las mismas características de la población meta, así como cuatro de los expertos, comentaron que la palabra "formas" no contaba con criterios de claridad adecuados, ante lo cual se decidió cambiar esta palabra por la de actividades teniendo en cuenta la recomendación de la filóloga, apoyado además en los fundamentos conceptuales que Orem (1983) realiza del autocuidado, definido como "la práctica de actividades que las personas inician y hacen por sí mismas para el mantenimiento de la vida, la salud y el bienestar" (p.55). Partiendo de esta premisa, ella visualiza esta acción como un conjunto de actividades que el individuo realiza para tener un estado adecuado de salud y bienestar y por esta razón. Por tanto, se modificó el ítem 2 de la siguiente manera: reviso si las actividades que practico habitualmente para mantenerme con salud son buenas.

Ítem 7. "Yo puedo buscar mejores formas para cuidar mi salud que las que tengo ahora".

Tanto la experta en filología, estadística y una enfermera, así como una de las personas de la muestra coincidieron en que la palabra formas era ambigua y no dejaba en claro a qué se refiere. Por este motivo, se decidió modificarla, de la siguiente manera: "yo puedo buscar mejores acciones para cuidar mi salud que las que tengo ahora," lo cual no modifica su fundamentación teórica ya que como Orem (1983) menciona; "el autocuidado tiene su razón de ser. Es la acción que tiene un modelo y un desarrollo y, cuando se hace eficazmente, contribuye de forma específica a la integridad estructural, funcionamiento y desarrollo humano" (p.55). Tal como se muestra, Orem considera el autocuidado como una acción. Con esta adaptación del ítem se establece una redacción más clara y comprensible para las personas, se elimina la ambigüedad existente, sin perder lo que se pretende medir, es decir, si la persona logra buscar acciones concretas para cuidar la salud de las que ya se tienen. 

de ser".

Ítem 10. "Cuando hay situaciones que me afectan yo las manejo de manera que pueda mantener mi forma

La expresión "mi forma de ser" no contaba con criterios de claridad suficientes, pues -al parecer de las expertas en enfermería- es un término subjetivo. Sin embargo, al analizarlo a la luz de la teoría de autocuidado, Orem (1983), hace mención a los ocho requisitos universales de autocuidado en lo que respecta a la promoción de la normalidad, las acciones para satisfacer dicho requisito se enfocan a la importancia y necesidad de conservar la integridad personal o forma de ser de cada individuo ante cualquier situación que le permita estar en bienestar, mediante acciones como mantener un concepto realista de cada uno, actuando de manera tal que se logre mantener la integridad estructural y funcionamiento de cada uno, y teniendo la capacidad de lograr identificar cualquier anomalía o alteración de la estructura y funcionamiento de uno mismo. Es por esta razón que no se le realizó ninguna modificación ya que posee sustento a la luz de la teoría.

\section{- $\quad$ Validación de contenido}

La prueba de Kaiser-Meyer-Olkin (KMO) dio un índice de 0,65, dato que resulta ser aceptable; la prueba de esfericidad de Bartlett dio estadísticamente significativa con un valor de p<0,000; 276 gl y un Chi de 563,9, lo cual favoreció el análisis para lograr determinar los factores subyacentes de la matriz de correlación (Martínez, $\underline{2011)}$.

El estudio factorial mediante el método ACP con rotación Varimax sugirió el análisis con 5 ejes factoriales que explicaron el 56,76\% de la varianza total (tabla 1), tomando como criterio asignar un ítem al que presentara la mayor carga factorial dentro de estos ejes.

Factor 1 (F1): 16,89\% de la varianza total, incluyó los ítems 1, 2, 9, 10, 13, 15 y 23. En este eje factorial predominaron los ítems que pertenecen a la categoría de prevención de peligros contra la vida, el funcionamiento y el bienestar humano. Al respecto Orem (1983) hace mención de un tipo de prevención secundaria fundamental luego de que comienza una enfermedad y que está orientada a evitar la aparición de complicaciones e incapacidad prolongada que se vincula a las características de este grupo poblacional. Seguidamente se presentaron los requisitos de promoción del funcionamiento y desarrollo humano dentro de grupos sociales, los cuales según Orem (1995) contribuyen al mantenimiento integral del ser humano, ya que el prevenir peligros como los relacionados a una enfermedad crónico, se promueve el funcionamiento y bienestar de la persona que la padece. Además, este factor incluyó dos ítems relacionados con los requisitos universales de alimentación y los de equilibrio entre actividad y descanso. Orem (1983), a su vez, hace mención al requisito de alimentación como uno de los suministros indispensables para el metabolismo y la producción de energía, en lo que juega un papel fundamental el balance existente entre la actividad y el descanso, ya que de ello dependerá el control de la energía voluntariamente utilizada, la regulación de estímulos del ambiente y la sensación de bienestar general de la persona.

- $\quad$ Factor 2 (F2): 15,32\% de la varianza total e incluyó los ítems 3, 14, 17, 18, 19, 21, 22 y 24 de la escala. Fue el factor que más ítems incorporó, cuya mayoría correspondió al requisito universal de autocuidado de prevención de peligros contra la vida, el funcionamiento y el bienestar humano como lo sucedido en el factor anterior; incluyó además requisitos de promoción del funcionamiento y desarrollo humano dentro de grupos sociales, equilibrio entre actividad y descanso y equilibrio entre soledad y comunicación social, éste ultimo 


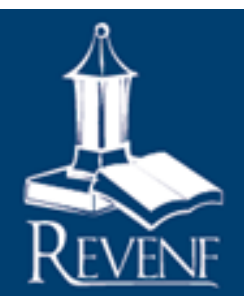

concerniente, según Orem (1995), a aquella condición esencial que debe ser proporcionada para un adecuado desarrollo, ya que ambos aspectos se complementan para alcanzar la reflexión personal de la mano de la adquisición de la cultura y obtención de recursos necesarios para la vida.

- $\quad$ Factor 3 (F3): 10,29\% de la varianza total e incluyó, los ítems 4, 5, 6, 11, 16 y 20, de los cuales solo, se presentaron dos requisitos en igual proporción: prevención de peligros contra la vida, el funcionamiento y el bienestar humano, y equilibrio entre actividad y descanso.

- $\quad$ Factor 4 (F4): 7,33\% de la varianza total e incluye los ítems 7 y 12 referentes a los requisitos de prevención de peligros contra la vida, el funcionamiento y el bienestar humano y equilibrio entre interacción social y soledad.

- $\quad$ Factor 5 (F5): 6,91\% de la varianza total e incluye el ítem de la escala número 8 concerniente al requisito de prevención de peligros contra la vida, el funcionamiento y el bienestar humano. 
Tabla 1.

Matriz de cargas factoriales para los ejes factoriales según análisis de componentes principales y rotación varimax para la población con diagnóstico de enfermedad crónica, 2011. $(\mathrm{n}=106)$

\begin{tabular}{|c|c|c|c|c|}
\hline & \multicolumn{4}{|c|}{ Factor } \\
\hline & 1 & 2 & 3 & 4 \\
\hline $\begin{array}{l}\text { 10. Cuando hay situaciones que me afectan yo las manejo de manera que } \\
\text { pueda mantener mi forma de ser }\end{array}$ & ,798 & 039 &,- 067 & 059 \\
\hline $\begin{array}{l}\text { 2. Reviso si las actividades que practico para mantenerme con salud son } \\
\text { buenas }\end{array}$ &, 783 & 153 &, 329 & ,151 \\
\hline 23. Puedo sacar tiempo para mí & ,749 & ,208 & ,023 &, 050 \\
\hline $\begin{array}{l}\text { 9. Para mantener el peso que me corresponde hago cambios en mis hábitos } \\
\text { alimenticios }\end{array}$ & ,648 & ,326 &, 115 &,- 093 \\
\hline 15. Yo examino mi cuerpo para ver si hay algún cambio &, 596 & 145 & ,072 & ,277 \\
\hline $\begin{array}{l}\text { 1.A medida que cambian las circunstancias, yo voy haciendo ajustes para } \\
\text { mantener mi salud }\end{array}$ &, 536 &, 117 &, 486 &,- 070 \\
\hline 13. Puedo dormir lo suficiente como para sentirme descansado & ,356 & ,216 &,- 225 &,- 079 \\
\hline $\begin{array}{l}\text { 21. Si mi salud se ve afectada yo puedo conseguir la información necesaria } \\
\text { sobre qué hacer }\end{array}$ &,- 071 &, 757 &, 174 &,- 009 \\
\hline 22. Si yo no puedo cuidarme puedo buscar ayuda & ,106 & ,703 &,- 076 &, 340 \\
\hline $\begin{array}{l}\text { 17. Cuando tengo que tomar una nueva medicina, cuento con una persona que } \\
\text { me brinda información sobre los efectos secundarios }\end{array}$ & ,218 & ,696 & ,127 &,- 234 \\
\hline $\begin{array}{l}\text { 19. Soy capaz de evaluar qué tanto me sirve lo que hago para mantenerme con } \\
\text { salud }\end{array}$ &, 444 & 638 &,- 033 &,- 083 \\
\hline $\begin{array}{l}\text { 18. Soy capaz de tomar medidas para garantizar que mi familia y yo no } \\
\text { corramos peligro }\end{array}$ &, 144 &, 575 & ,203 &, 002 \\
\hline $\begin{array}{l}\text { 14. Cuando obtengo información sobre mi salud, pido explicaciones sobre lo } \\
\text { que no entiendo }\end{array}$ &, 341 &, 565 & , 158 & ,317 \\
\hline $\begin{array}{l}\text { 24. A pesar de mis limitaciones para movilizarme, soy capaz de cuidarme como } \\
\text { a mí me gusta }\end{array}$ & ,382 & ,484 &, 053 & ,000 \\
\hline $\begin{array}{l}\text { 3. Si tengo problemas para moverme o desplazarme, me las arreglo para } \\
\text { conseguir ayuda }\end{array}$ & ,183 & ,482 & ,237 & ,220 \\
\hline 6. Me faltan las fuerzas necesarias para cuidarme como debo &,- 102 &,- 004 & ,707 &, 018 \\
\hline 5. Hago en primer lugar lo que sea necesario para mantenerme con salud & ,172 & 190 & ,642 &,- 046 \\
\hline 4. Yo puedo hacer lo necesario para mantener limpio el ambiente donde vivo & ,043 & ,216 &, 587 &,- 025 \\
\hline $\begin{array}{l}\text { 20. Debido a mis ocupaciones diarias, me resulta difícil sacar tiempo para } \\
\text { cuidarme }\end{array}$ & ,403 & ,030 &, 516 &,- 084 \\
\hline $\begin{array}{l}\text { 16. He sido capaz de cambiar hábitos que tenía muy arraigados con tal de } \\
\text { mejorar mi salud }\end{array}$ & ,385 & ,383 & ,400 &,- 018 \\
\hline $\begin{array}{l}\text { 11. Pienso en hacer ejercicio y descansar un poco durante el día pero no llego } \\
\text { hacerlo }\end{array}$ & ,015 & ,269 & ,268 &,- 686 \\
\hline 12. Cuando necesito ayuda, puedo recurrir a mis amigos siempre & ,303 & 147 &,- 122 & ,658 \\
\hline $\begin{array}{l}\text { 7. Yo puedo buscar mejores acciones para cuidar mi salud que las que tengo } \\
\text { ahora }\end{array}$ &,- 178 & 145 & ,313 & ,625 \\
\hline 8. Cambio la frecuencia con que me baño para mantenerme limpio &,- 031 & 039 &,- 074 &,- 070 \\
\hline
\end{tabular}

Fuente: Cubillo, Porras, Ramírez, Sirias. 2011 
- Consistencia interna y confiabilidad

Los datos obtenidos resultan confiables, ya que arrojaron un valor del Alfa de Cronbach de 0,84 (84\%) 1o que significa que el total de los ítems que contempla la escala logran medir un mismo concepto o se correlacionan positivamente entre sí, independientemente del tipo de contenido (Ruíz, 2011).

La tabla siguiente muestra que el ítem 8 de la escala, con respecto a la correlación elemento-total, da un resultado negativo: si este se eliminara, el Alfa de Cronbach aumentaría un 0,016 y queda un total final de 0,860 (86\%) y así un grado de confiabilidad aún mayor; sin embargo, no afecta su conservación ya que los valores recomendados para estos índices están entre 0.7 y 0.9 (70\% y 90\%) (Campo y Oviedo, 2008)

\section{Tabla 2.}

Medición de la confiabilidad global y de cada ítem de la escala ASA, adaptada para la población costarricense, 2011.

\begin{tabular}{|c|c|c|c|c|}
\hline Ítem & $\begin{array}{c}\text { Media de la escala } \\
\text { si se elimina el } \\
\text { elemento }\end{array}$ & $\begin{array}{c}\text { Varianza de la } \\
\text { escala si se } \\
\text { elimina el } \\
\text { elemento }\end{array}$ & $\begin{array}{l}\text { Correlación } \\
\text { elemento-total } \\
\text { corregida }\end{array}$ & $\begin{array}{c}\text { Alfa de Cronbach si se } \\
\text { elimina el elemento }\end{array}$ \\
\hline 1. & 74,73 & 74,332 & 649 & ,830 \\
\hline 2. & 74,85 & 72,515 & ,636 & ,828 \\
\hline 3. & 74,96 & 76,402 & ,331 & 841 \\
\hline 4. & 74,30 & 79,268 & ,410 & ,839 \\
\hline 5. & 74,70 & 76,422 &, 514 & ,835 \\
\hline 6. & 74,66 & 79,458 & ,214 & ,844 \\
\hline $\begin{array}{l}7 . \\
8 .\end{array}$ & $\begin{array}{l}74,98 \\
74,97\end{array}$ & $\begin{array}{l}79,327 \\
83,143\end{array}$ & $\begin{array}{l}, 202 \\
-, 070\end{array}$ & $\begin{array}{l}, 845 \\
, 861\end{array}$ \\
\hline $\begin{array}{l}9 . \\
10 .\end{array}$ & $\begin{array}{l}75,22 \\
74,78\end{array}$ & $\begin{array}{l}75,173 \\
78,057\end{array}$ & $\begin{array}{l}, 433 \\
, 351\end{array}$ & $\begin{array}{l}, 837 \\
, 840\end{array}$ \\
\hline 11. & 75,50 & 81,041 & ,056 & ,853 \\
\hline 12. & 74,83 & 76,451 & ,343 & 840 \\
\hline 13. & 74,92 & 76,110 & ,388 & ,838 \\
\hline $\begin{array}{l}14 . \\
15 .\end{array}$ & $\begin{array}{l}74,47 \\
74,76\end{array}$ & $\begin{array}{l}75,828 \\
74,414\end{array}$ & $\begin{array}{l}, 567 \\
, 560\end{array}$ & $\begin{array}{l}, 833 \\
, 832\end{array}$ \\
\hline $\begin{array}{l}16 . \\
17 .\end{array}$ & $\begin{array}{l}74,75 \\
74,90\end{array}$ & $\begin{array}{l}75,150 \\
74,056\end{array}$ & $\begin{array}{l}, 551 \\
, 467\end{array}$ & $\begin{array}{l}, 833 \\
, 835\end{array}$ \\
\hline 18. & 74,38 & 78,392 & ,440 & ,838 \\
\hline 19. & 74,62 & 76,238 & ,557 & ,834 \\
\hline 20. & 75,21 & 75,590 & ,386 & ,839 \\
\hline 21. & 74,54 & 77,924 & ,482 & ,837 \\
\hline 22. & 74,63 & 76,216 & ,498 & ,835 \\
\hline 23. & 74,80 & 73,315 & ,602 & 830 \\
\hline 24. & 74,64 & 76,368 & ,476 & ,835 \\
\hline
\end{tabular}

Fuente: Cubillo, Porras, Ramírez, Sirias. 2011 


\section{DISCUSIÓN}

Como parte de un cambio en los estilos de vida de una población en desarrollo, la Organización Panamericana de la Salud (2004) se refiere a un proceso conocido como "transición epidemiológica", en el cual se ha dado un desplazamiento de la mortalidad a grupos de edad más avanzada y un cambio en las causas de muerte con un predominio de las enfermedades crónicas no transmisibles, debido a la aparición de factores de riesgo relacionados con el proceso de desarrollo y urbanización, la disminución de la fecundidad y el mejoramiento de las tasas de letalidad, lo cual explica que desde el siglo pasado las enfermedades crónicas han pasado a ser la principal causa de muerte, dejando atrás las enfermedades transmisibles como el dengue, la tuberculosis, la hepatitis, entre otras.

En el año 2006, el Ministerio de Salud de Costa Rica señaló que estas enfermedades se han constituido en uno de los principales problemas de salud pública, datos que respalda el Instituto Nacional de Estadísticas y Censos (INEC, 2008), entidad que indica que en dicho año fallecieron alrededor de 8626 personas, debido a enfermedades cardiovasculares y el cáncer o sea, el $51,1 \%$ de la totalidad de los fallecimientos que se registran en el territorio nacional (Hernández, 2010).

Ante este panorama de la epidemiología de la salud costarricense, fue necesario validar la escala ASA con personas que padecen una patología crónica, ya que es de interés del profesional en ciencias de la salud conocer el autocuidado de este creciente grupo y las estrategias de intervención para disminuir los efectos negativos, a pesar de evidencias acerca de las dificultades que enfrentan las personas con algún déficit de autocuidado para modificar conductas nocivas (Flores y Gómez, 2008).

En este sentido, Fernández y Manrique-Abril (2010), apuntan que el profesional de enfermería juega un papel importante en la maximización de la independencia de toda persona, especialmente si se trata de un cuidado dependiente en el que se debe capacitar no solo al individuo, sino también involucrar a la familia y la comunidad en el cuidado de su salud mediante el fomento de hábitos de vida saludables. De igual forma en el estudio realizado por Rivera y Díaz (2007), se señala que, para el profesional de enfermería, las indagaciones científicas orientadas a valorar la capacidad de agencia de autocuidado en personas con alguna desviación de la salud es una herramienta de gran utilidad para la promoción de la salud y prevención de enfermedades en aquellos aspectos que pueden generar un deterioro en la calidad de vida que a futuro implicarán un elevado costo socioeconómico y que van a requerir un abordaje integral en el proceso de atención por parte de dicha disciplina, fin implícito que se busca conseguir con el uso de la escala ASA ya validada para Costa Rica.

Orem (1983) menciona que una persona que presenta alguna alteración del estado de salud debe realizar modificaciones y adaptaciones necesarias para poder cumplir con los requisitos universales de autocuidado y mantener de manera adecuada la vida, la salud y el bienestar. Al respecto, según Orem se destaca "las características de los cambios de salud como procesos de larga duración determinan los tipos de demandas de cuidado que los individuos experimentan mientras viven con los efectos de procesos patológicos o anormales y que sobrepasan la duración del proceso" (1983. p.73). La definición anterior coincide de manera oportuna con las características de una enfermedad crónica en la cual sus manifestaciones se hacen presentes en un periodo de duración prolongada que implica una modificación y adaptación a nuevos estilos de vida que permitan poner en práctica un adecuado proceso de autocuidado. 
Como se evidenció con esta investigación, la mayoría de personas presentaron hipertensión arterial (HTA) como la enfermedad más común, lo cual es confirmado por datos de la Organización Panamericana de la Salud que indica que 600 millones de personas actualmente la padecen a nivel mundial, lo que la convierte en una de las primeras causas de mortalidad, datos que quedan evidenciados con estadísticas del año 2007, cuando en América ocurrieron 1.5 millones de muertes por enfermedades cardiovasculares, de los cuales 662011 personas tuvieron una cardiopatía isquémica y 336 809, una enfermedad cerebrovascular (OPS, 2011).

Esta problemática genera una discapacidad que resulta en una carga pesada para los individuos, las familias y el sistema de salud, por lo cual no es de extrañar que muchas de las investigaciones tengan como grupo meta individuos que tienen HTA, así por ejemplo, la mayoría de estudios en los cuales se ha medido la capacidad de agencia de autocuidado de alguna población, así como la validación de la escala ASA en distintos países, se han elaborado con poblaciones de personas diagnosticadas con hipertensión arterial o alguna enfermedad crónica cardiovascular (Achury et al, 2008; Velandia y Rivera 2009; Arredondo, 2010).

En Costa Rica, con los resultados de la validación de apariencia, contenido y consistencia interna, la escala ASA demuestra que es una herramienta que puede ser utilizada con personas diagnosticadas con alguna enfermedad crónica en cualquier nivel de atención de salud, ya que determinó la pertinencia cultural y social del lenguaje utilizado para hacer un uso confiable y permitir a la profesión una mayor autonomía y capacidad de trabajo multidisciplinar, interdisciplinar y transdisciplinar en la atención brindada a los usuarios del sistema de salud costarricense, ya que hace valer un criterio científico sustentado en una teoría propia y tan importante como la Teoría del Autocuidado de Dorothea Orem.

\section{CONCLUSIÓN}

Los resultados del proceso de validación de apariencia permitieron realizar modificaciones y adaptaciones a la escala en aras de lograr una mejor comprensión.

La inclusión de personas adultas con diagnóstico de enfermedad crónica permite evaluar -con la escala ASA- su capacidad de agencia de autocuidado, generar diagnósticos propios de la disciplina y determinar estrategias de intervención. El análisis factorial arrojó 5 ejes factoriales y logró afirmar que el instrumento cuenta con una adecuada validez de contenido, con propiedades psicométricas válidas y adecuadas. Se demostró una alta confiabilidad tras la obtención de un coeficiente Alfa de Cronbach de 0,84, lo que refleja que los 24 miden un mismo concepto.

En Costa Rica, enfermería puede contar con un instrumento válido y confiable para medir la capacidad de agencia de autocuidado en las personas con diagnóstico de enfermedad crónica que se encuentran en la etapa de la adultez.

\section{Conflicto de intereses.}

Las autoras declaran que no existe conflicto de intereses en el desarrollo de este estudio. 


\section{REFERENCIAS BIBLIOGRAFICAS}

Achury, S., Sepúlveda, G. y Rodríguez, S. (2008). Validez de Apariencia y Contenido de un instrumento para evaluar la Capacidad de Agencia de Autocuidado en el paciente con hipertensión Arterial. Colombia. Red de Revistas cientificas de América Latina y el Caribe, España y Portugal. (Versión en línea). Recuperado de: http://redalyc.uaemex.mx/src/inicio/ArtPdfRed.jsp?iCve=145212853007

Achury, S., Sepúlveda, C. y Rodríguez, S. (2008). Capacidad de Agencia de Autocuidado en personas con hipertensión arterial. Revista de Enfermería. (Versión en línea). Recuperado de: file:///C:/Documents\%20and\%20Settings/Jean/Mis\%20documentos/SEMINARIO\%20DE\%20GRADUACION/Temaslibres capacidaddeagenciadeautocuidado.htm

Álvarez, R. (1994). Estadística multivariante y no paramétrica con SPSS. Madrid: Díaz de Santos. (Versión en línea). Recuperado de: http://books.google.co.cr/books?id=GxhpROT-

HB0C\&pg=PA237\&dq=an\%C3\%A1lisis + factorial + rotaci $\% \mathrm{C} 3 \% \mathrm{~B} 3 \mathrm{n}+$ Varimax $\& 1 \mathrm{r}=\# \mathrm{PPP} 1, \mathrm{M} 1$

Arredondo, E. (2010). Comportamiento y capacidad de agencia de autocuidado en adultos con insuficiencia cardiaca congestiva. Universidad Nacional de Colombia. Revista Avances en Enfermería, XXVIII (1). (Versión en línea). Recuperado de: http://www.enfermeria.unal.edu.co/revista/articulos/xxviiil 3.pdf

Campo, A. y Oviedo, H. (2008). Propiedades psicométricas de una escala: la consistencia interna. Revista Salud Pública, 10. (Versión en línea). Recuperado de: http://www.scielosp.org/scielo.php?pid=S012400642008000500015\&script=sci_abstract

Contreras, A., Flórez, I. y Herrera, A. (2008). Un instrumento para evaluar la adherencia: su validez facial y confiabilidad. Cartagena. (Versión en línea). Recuperado de: http://www.enfermeria.unal.edu.co/revista/articulos/xxvi2_4.pdf

Evers, G. (1998). Meten van zelfzorg. Verpleegkundige instrumenten. Voor onderzoek en klinische praktijk. Leuven University Press.

Fernández, A. y Manrique-Abril, F. (2010). Efecto de la intervención educativa en la agencia de autocuidado del adulto mayor hipertenso de Boyacá. Ciencia y Enfermería XVI (2). (Versión en línea). Recuperado de: http://www.scielo.cl/pdf/cienf/v16n2/art_09.pdf

Flores, G. B. y Gómez, O. L. (2008). Capacidad de agencia de autocuidado en pacientes con hipertensión arterial. Facultad de Enfermería, Universidad Veracruzana, México. Tesis para optar por El grado de Licenciatura en Enfermería. (Versión en línea). Recuperado de: http://cdigital.uv.mx/bitstream/12345678/626/1/TESIS\%20FINAL.pdf

George, D. \& Mallery, P. (1995). SPSS/PC + Step by: A simple guide and reference. Belmont, CA: Wadsworth Publishing Company.

Hernández, R, Fernández, C. y Baptista, P. (2006). Metodología de la Investigación. 4ta ed. Ed. Mc-Graw Hill Interamericana. México, DF.

Hernández, J. (2010). Avances en promoción de la salud y prevención de las enfermedades crónicas en Costa Rica. Revista costarricense de Salud Pública 19(1): 48-55. (Versión en línea) Recuperado de: http://www.scielo.sa.cr/scielo.php?script=sci_arttext\&pid=S1409-14292010000100009\&lng=es. 
Instituto Nacional de Estadística y Censos de Costa Rica (2008). Panorama Demográfico 2008. Recuperado de: http://www.inec.go.cr/Web/Home/pagPrincipal.aspx

Kerlinger, F. y Lee, H. (2002). Investigación del comportamiento. Métodos de investigación en Ciencias Sociales. México D.F.: McGraw-Hill.

Lamprea, J. y Gómez, C. (2007). Validez en la Evaluación de Escalas. Revista Colombiana de Psiquiatría. Asociación Colombiana de Psiquiatría. Bogotá, Colombia. (Versión en línea). Recuperado de: http://redalyc.uamex.mx

Leiva, V. (2009) ¿Por qué enseñar bioética social y de salud pública en Enfermería? Revista Enfermería Actual en Costa Rica. $\mathrm{N}^{\circ}$ 17. (Versión en línea) Recuperado de:

http://redalyc.uaemex.mx/redalyc/html/448/44812828001/44812828001 $1 . \mathrm{html}$

Manrique-Abril, F., Fernández, A. y Velandia, A. (2009). Análisis factorial de la escala Valoración de Agencia de Autocuidado (ASA) en Colombia. Revista Aquichan 9 (3).

Martínez, E. (2011). Análisis Factorial. 2da. Canarias: Editorial Contreras.

Ministerio de Salud (2006). Observatorio de politicas de enfermedades no transmisibles. Costa Rica (versión en línea). Recuperado de: http://www.ministeriodesalud.go.cr/index.php/gestores-salud-tecno-ciencia-encuetas-ms/doc details/392observatorio-de-politicas-de-enfermedades-no-transmisibles-estudio-de-caso

Monge, Ch. (2004). Comparación de la capacidad de agencia de autocuidado en dos grupos de mujeres adultas: uno de ellos con hipertensión arterial y el otro con enfermedad cardiovascular en el Hospital Mario Catarino Rivas, San Pedro Sula. Honduras. Universidad Nacional de Colombia. Bogotá.

Morales, P. (2011). El Análisis Factorial en la construcción e interpretación de test, escalas y cuestionarios. Universidad Pontífica Comillas. Madrid.

Orem, D. (1983). Normas prácticas en Enfermería. Madrid: Ediciones Pirámide.

Orem, D. (1995). Nursing: Concepts of practice. Fifth edition. Missouri: Mosby.

Organización Mundial de la Salud. (2008) Temas de salud: enfermedades crónicas. (Versión en línea). Recuperado de: http://www.who.int/topics/chronic diseases/es/

Organización Panamericana de la Salud (2004). Perfil del sistema de servicios de salud de Costa Rica* / Organización Panamericana de la Salud, Ministerio de Salud. San José, Costa Rica. (Versión en línea). Recuperado de: http://www.bvs.sa.cr/php/situacion/perfil.pdf

Organización Panamericana de la Salud (2011). Consulta regional: prioridades para la salud cardiovascular en las Américas. Mensajes claves para los decisores. Washington, D. C. 
Ortiz, G. (2002). Factores básicos condicionantes del autocuidado asociados a la capacidad potencial de autocuidarse para promoción de la salud de personas de 18 años y más, que viven en situación de enfermedad cardiovascular y que asisten a consulta al centro de salud Pedro Altamirano, Nicaragua. Universidad Nacional de Colombia. Bogotá.

Papalia, D. (2005). Desarrollo Humano. Novena Edición. México D. F.: Mc-Graw Hill.

Porras, M. (2002) Factores básicos condicionantes del autocuidado asociados a la capacidad potencial de autocuidarse y promover la salud de personas de 18 años y más, que viven en situación de enfermedad cardiovascular y asisten a la consulta externa del Hospital Roosevelt en la ciudad de Guatemala. Universidad Nacional de Colombia. Bogotá.

Real Academia Española (2011) Diccionario de la lengua española. Recuperado de: http://www.rae.es/rae.html

Rivera, A. y Díaz, L. (2007). Relación entre la capacidad de agencia de autocuidado y los factores de riesgo cardiovascular. Colombia. Revista Cuadernos Hospital de Clínicas, 52 (2). (Versión en línea). Recuperado de: http://www.docentes.unal.edu.co/lnriveraa/docs/RELACION\%20ENTRE\%20LA\%20CAPACIDAD\%20DE\%20AGENCIA \%20DE\%20AUTOCUIDADO \%20Y\%20LOS\%20FACTORES\%20DE\%20RIESGO\%20CARDIOVASCULAR.pdf

Rivera, A. (2006). Capacidad de Autocuidado en personas con hipertensión arterial hospitalizadas en una clínica de Bogotá, Colombia. Revista de Salud Pública, 8(3).

Rodríguez, M. y Díaz, R. (2011). Instrumento para medir las capacidades de autocuidado en los pacientes adultos hospitalizados con terapia intravenosa. (Versión en línea) Diversidad Cultura y Salud. Recuperado de:

http://www.eumed.net/libros/2011f/1142/instrumento_para_medir_las_capacidades_de_autocuidado_en_los_pacientes_adul tos_hospitalizados_con terapia_intravenosa.html

Ruiz, C. (2011). Confiabilidad. Universidad Pedagógica Experimental Libertador. Maracaibo. (Versión en línea). Disponible en: http://investigacion.upeu.edu.pe/images/7/74/Validez.pdf

Sánchez, R. y Echeverri, J. (2004). Validación de escalas en medición de salud. Revista de Salud Pública, Bogotá.

Velandia, A. y Rivera, A. (2009). Confiabilidad de la escala de "Apreciación de la agencia de autocuidado (ASA) segunda versión en español adaptada para población colombiana”. Revista avances en enfermería, 27 (1). 